\title{
Response to Checkpoint Inhibitor Therapy in Advanced Classic Kaposi Sarcoma: A Case Report and Immunogenomic Study
}

James Saller, MDª, Christine M. Walko, PharmDa; Sherri Z. Millis, $\mathrm{PhD}^{\mathrm{c}}$; Evita Henderson-Jackson, MD; Rikesh Makanji, MD'; and Andrew S. Brohl, MD ${ }^{\mathrm{e}, \mathrm{f}}$

\begin{abstract}
Kaposi sarcoma (KS) is an uncommon angioproliferative malignancy that is associated with human herpesvirus 8 . Although there has been recent enthusiasm for evaluating immune checkpoint inhibition as a therapeutic option for viral-associated tumors, the clinical utility in this disease is currently unknown. We report a case of advanced classic KS refractory to multiple lines of chemotherapy that experienced a partial response to anti-PD-1 therapy. Comprehensive molecular profiling was performed on a diagnostic tumor biopsy sample. Molecular profiling data from 8 additional male patients with KS were reviewed and compared with those of the index case. The genomic profile of the index case was notable for higher-than-typical somatic mutational burden, including pathogenic mutation in multiple well-described cancer genes, such as TP53, CDKN2A, NOTCH1, and KRAS. Our case suggests that further clinical study of checkpoint inhibitor therapy in classic KS is warranted, and provides a hypothesis for future immunogenomic biomarker analysis in this disease.
\end{abstract}

J Natl Compr Canc Netw 2018;16(7):797-800 doi: 10.6004/jncen.2018.7018

\section{Background}

Kaposi sarcoma (KS) is an uncommon angioproliferative malignancy that is associated with human herpesvirus 8 (HHV-8). KS is most commonly observed in patients affected by HIV/AIDS, and is one of several AIDS-defining illnesses. It has also been observed in association with chronic immunosuppression, such as in the solid organ transplant population. The remaining nonimmunosuppressed and nonendemic cases of classic KS are particularly rare. ${ }^{1}$

Most cases of classic KS are indolent, typified by cutaneous lesions of the lower extremities. For advanced cases, chemotherapy is often administered, with reasonably high response rates for a variety of agents. ${ }^{2} \mathrm{Pe}$ gylated liposomal doxorubicin, approved by the FDA for AIDS-related KS, is often used as first-line therapy,

aPersonalized Medicine Institute and bepartment of Anatomic Pathology, Moffitt Cancer Center, Tampa, Florida; 'Foundation Medicine, Inc., Cambridge, Massachusetts; and dDepartment of Radiology, 'Sarcoma Department, and ${ }^{\mathrm{f} C h e m i c a l ~ B i o l o g y}$ and Molecular Medicine Program, Moffitt Cancer Center, Tampa, Florida.

Submitted October 4, 2017; accepted for publication February 20, 2018. Dr. Walko has received honoraria from Bristol-Myers Squibb and Merck. with major responses noted in most patients. $^{3}$ For patients nonresponsive to chemotherapy, particularly if they are resistant to several lines of chemotherapy, optimal management is not known.

There has been recent enthusiasm for evaluating immune checkpoint inhibition as a therapeutic option for viral-associated tumors, due in part to the presence of viral neoantigens that may prove to be immunogenic. Immunotherapy clinical trials that include HIV-associated KS are ongoing (ClinicalTrials.gov identifiers: NCT02595866, NCT02408861). Recent studies have demonstrated significant PD-1/PD-L1 expression in tumors and the microenvironment in both HIV-associated and classic KS, further supporting the investigation of agents targeting these molecules in this disease. ${ }^{4,5}$

Dr. Millis is an employee of Foundation Medicine. The other authors have disclosed that they have no financial interests, arrangements, affiliations, or commercial interests with the manufacturers of any products discussed in this article or their competitors.

Correspondence: Andrew S. Brohl, MD, Sarcoma Department, Moffitt Cancer Center, 12902 Magnolia Drive, Tampa, FL 33612.

Email: andrew.brohl@moffitt.org 
Saller et al

Interest in investigating immunotherapy in classic KS has resulted in a planned phase II study of nivolum$\mathrm{ab}$ and ipilimumab in this disease (ClinicalTrials.gov identifier: NCT03219671), but currently there is a paucity of clinical data regarding the efficacy of this approach. Furthermore, not much is known about pertinent immunogenomic features of $\mathrm{KS}$, such as tumor mutational burden (TMB). We therefore present a case of advanced classic KS treated successfully with PD-1 inhibitor therapy. Next-generation sequencing (NGS)-based molecular testing was performed on a pretreatment tumor sample from this case. We review the clinical and immunogenomic findings in this patient, and further review the genomic profile of $8 \mathrm{ad}$ ditional cases of KS to speculate on the broader applicability of our clinical experience. To our knowledge, this is the only reported case of successful immune checkpoint inhibitor (ICI) treatment of classic KS, and the most extensive evaluation of NGS-based tumor genomic profiling of KS in the literature to date.

\section{Case Presentation}

A 75-year-old homosexual African-American man with a history of hypertension, hyperlipidemia, and atrial fibrillation presented with a painless, slowly growing mass in his suprapubic region over a period of approximately 6 months. Concern for malignancy on clinical examination led to radiologic evaluation with PET/CT, which showed the mass to be highly metabolically active (standardized uptake value, 31.8) and revealed extensive PET-avid right inguinal and pelvic lymphadenopathy. He underwent an excisional biopsy and pathologic evaluation of the suprapubic mass. Histologic evaluation revealed morphology described as a nodular-like proliferation of spindle-shaped cells in cellular bundles and fascicles with marked nuclear atypia and cellular pleomorphism with numerous slitlike spaces containing erythrocytes (Figure 1). Areas of necrosis, a prominent admixed inflammatory infiltrate of lymphocytes and plasma cells, and frequently extravasated erythrocytes and hemosiderin deposits were seen. Immunohistochemistry was performed, which stained positive for vimentin, CD31, CD34, D2-40, and strong, diffuse nuclear staining of HHV8. The Ki-67 was evaluated at $>90 \%$. These findings were interpreted as consistent with KS.

Of note, the patient reported that he was diagnosed with KS of the lower extremities by a derma-

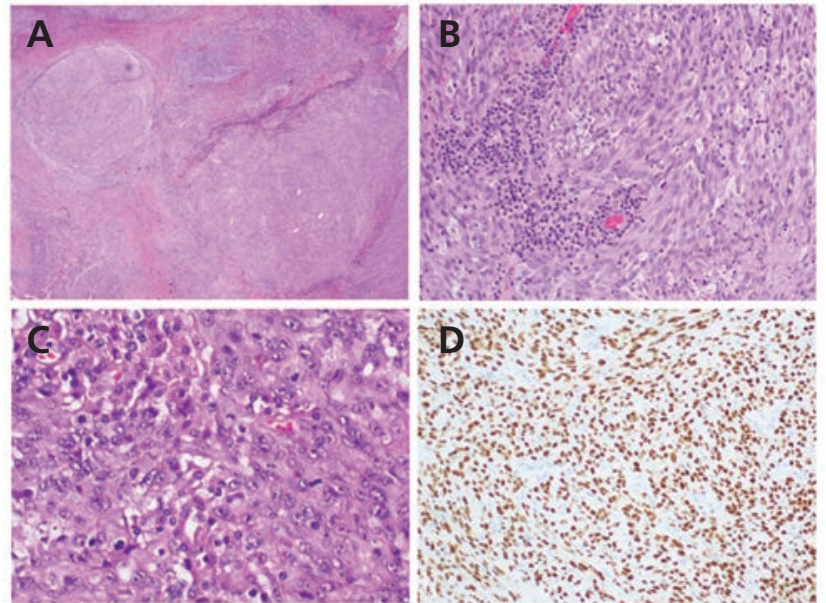

Figure 1. Tumor specimen pathologically consistent with Kaposi sarcoma. (A) Low-power view illustrating the nodular architecture of the tumor (hematoxylin-eosin [HE], original magnification $\times 2$ ). (B) Cellular bundles and fascicles of spindle cells with associated inflammatory cells and occasional extravasated RBCs ( $\mathrm{HE}$, original magnification $\times 20$ ). (C) Area of lesional cells demonstrating marked nuclear atypia and mitoses with associated small vascular spaces ( $\mathrm{HE}$, original magnification $\times 40)$. (D) Strongly positive nuclear staining for human herpesvirus 8 ( $\mathrm{HE}$, original magnification $\times 20$ ).

tologist approximately 10 years previously after biopsy of a darkened skin area. Over time, he developed slowly progressive bilateral lower extremity edema, but no significant progression of obvious/discrete skin lesions, and he did not pursue any further evaluation or therapy for this finding. HIV testing was performed and was negative. He has no significant history of immunosuppression or opportunistic infections. He denied a history of steroid use, including topical steroids. He was a lifetime nonsmoker.

Given the advanced nature of his KS, the patient was started on systemic chemotherapy with liposomal doxorubicin $\left(20 \mathrm{mg} / \mathrm{m}^{2}\right.$ every 21 days $)$, but experienced disease progression after 6 cycles. Progression was indicated by tumor growth of all lymph node (LN) areas, such as a pelvic LN conglomerate that measured $4.4 \times 3.9 \mathrm{~cm}$ compared with $3.8 \times 3.4$ $\mathrm{cm}$ at baseline. He was then treated with second-line chemotherapy with weekly paclitaxel $\left(80 \mathrm{mg} / \mathrm{m}^{2}\right.$ on days 1,8 , and 15 of a 28 -day cycle), but experienced further disease progression after 3 cycles. Progression was indicated by further growth of all $\mathrm{LN}$ areas, such as the pelvic $\mathrm{LN}$ conglomerate now measuring 6.8 $\mathrm{x} 4.7 \mathrm{~cm}$ and the development of a newly apparent $1.8-\mathrm{cm}$ splenic lesion.

Given the lack of standard treatment options after progression on chemotherapy, commercial ge- 
nomic profiling was performed on his prior (pretreatment) biopsy specimen to evaluate for potential options. Notably, this testing reported 7 mutations/ $\mathrm{Mb}$, which is an intermediate TMB on this assay (defined as 6-19 mutations/ $\mathrm{Mb}$ ). In other tumor types, an intermediate TMB (vs low mutation burden of $<6$ mutations/Mb) has been associated with increased likelihood of ICI therapy response. ${ }^{6,7}$ Given a lack of alternative options, along with this genomic finding, the patient was treated with pembrolizumab on a compassionate use basis.

Twelve weeks after starting treatment with pembrolizumab, a follow-up CT showed a significant reduction in tumor burden. Pembrolizumab was continued, and a follow-up CT after 6 additional weeks and at week 30 of therapy confirmed ongoing response (Figure 2), which would meet criteria for partial response using immune-related response criteria $(55.0 \%$ reduction in sum of bidirectional measurements of target lesions). Subsequent restaging imaging after 42 weeks of therapy showed necrosis and increased size of a right inguinal LNS $(6.5 \times 5.5 \mathrm{~cm}$ vs $4.3 \times 3.9 \mathrm{~cm}$ previously), but stability at other disease sites and no new areas of disease. Radiation therapy to this isolated site of progression was planned while he remains on anti-PD-1 therapy. At the time of writing, the patient has not experienced significant toxicities on immunotherapy, including no immune-related toxicity.

\section{Results}

Comprehensive genomic profiling was performed as previously described on the presystemic treatment excisional biopsy specimen from our patient. ${ }^{8}$ Mutational profiling on the same platform was performed on tumors from 8 additional male patients for which the diagnosis was listed as KS. HHV-8 viral antigen was detected by molecular profiling in all 9 samples, including the index patient. Of the 8 additional patients, 3 were documented as HIV-positive, whereas HIV status was not documented for the other 5 .

Molecular profiling results are summarized in Table 1. Notably, the tumor from the index case had the highest mutational burden of those tested. Additionally, oncogenic mutations were present in a number of well-described cancer genes in this case, including inactivating mutations in cell cycle genes (TP53, CDKN2A) and the signaling pathway gene NOTCH1, as well as hotspot mutations in classic

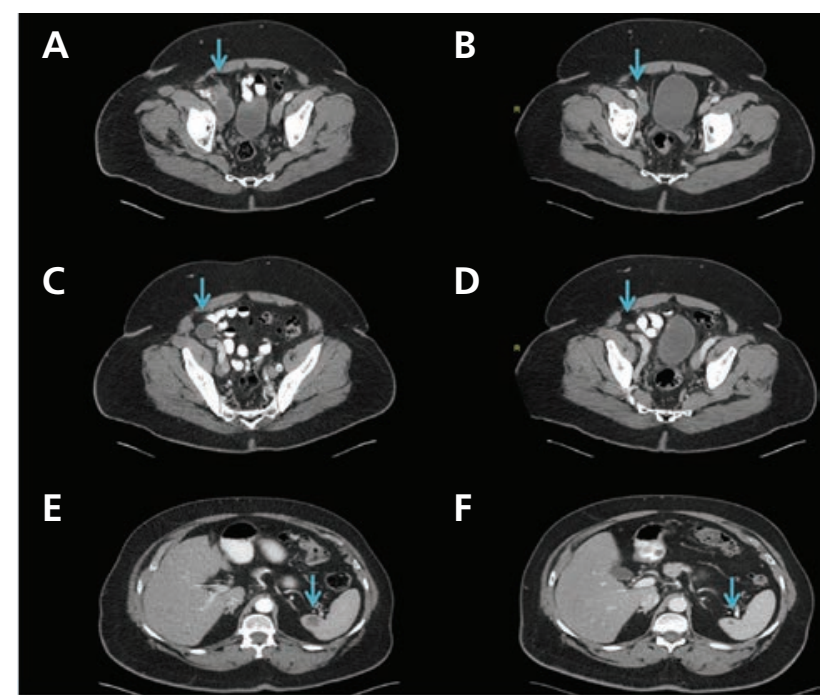

G

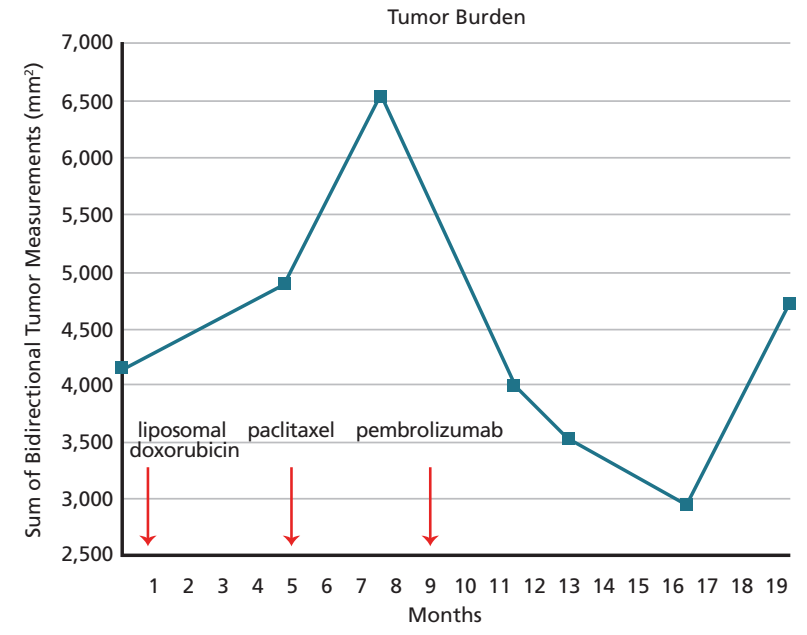

Figure 2. CT imaging ( $A, C, E)$ before and $(B, D, F)$ after 18 weeks of checkpoint inhibitor therapy with pembrolizumab demonstrate marked improvement of disease in the right external iliac chain and spleen. (G) Sum of bidirectional tumor measurements of target lesions demonstrate prior progression on chemotherapy followed by response to pembrolizumab therapy. Arrows indicate the timing of the start of a new systemic therapy.

oncogenes (KRAS, NRAS). In contrast, genomic findings in the other 8 tumors were limited mostly to variants of uncertain significance, although one additional tumor was affected by pathogenic TP53 and KRAS mutations. All tumors tested were microsatellite stable.

\section{Discussion and Conclusions}

$\mathrm{KS}$ is a rare soft tissue sarcoma that is uncommonly encountered outside of the immunosuppressed or HIV population. Although often an indolent disease, 
Saller et al

\begin{tabular}{|c|c|c|c|c|c|c|c|}
\hline Patient & Sex & Age, y & HIV Status & $\begin{array}{l}\text { Specimen } \\
\text { Location }\end{array}$ & $\begin{array}{l}\text { Mutational Burden } \\
\text { (Mutations/Mb) }\end{array}$ & $\begin{array}{l}\text { Significant } \\
\text { Alterations }\end{array}$ & Variants of Unknown Significance \\
\hline 1 & Male & 74 & Negative & Suprapubic mass & 7 & $\begin{array}{l}\text { CBL, CDKN2AIB (2), } \\
\text { KRAS, NOTCH1 (2), } \\
\text { NRAS, PIK3R1, TP53 (2) }\end{array}$ & $\begin{array}{l}\text { ARAF, BRCA1, CHD2, EPHA5, MAF, MKI67, } \\
\text { MLL3, NCOR1, NOTCH1, SPEN, TSC1 }\end{array}$ \\
\hline 2 & Male & 71 & Not reported & Right finger & 0 & & ARFRP1, XPO1 \\
\hline 3 & Male & 27 & Not reported & Skin & 0 & & HIST1H1C, MLH1, MSH3 (2), MTOR, TSC1 \\
\hline 4 & Male & 43 & Not reported & Skin & 1.7 & HNF1A & $\begin{array}{l}\text { HDAC7, MAPK1, MSH3, TYK2, WDR90, } \\
\text { YY1AP1 }\end{array}$ \\
\hline 5 & Male & 24 & Not reported & Stomach & 0 & & ARID2, MAP2K1, NCOR2 \\
\hline 6 & Male & 47 & Positive & Foot & 3.3 & & CPS1, ELP2, MKI67, MLH1, NCSTN \\
\hline 7 & Male & 64 & Not reported & Skin of leg & 0.8 & & CUX1, FANCD2, FGFR4, PTCH1, TYK2 \\
\hline 8 & Male & 61 & Positive & $\begin{array}{l}\text { Skin, } \\
\text { left inguinal fold }\end{array}$ & 3.3 & KRAS, TP53 & ASMTL, FGFR3, NCOR1, ROS1 \\
\hline 9 & Male & 43 & Positive & Skin, left leg & 2.5 & TLL2 & EP300, FANCL, FOXO3 \\
\hline
\end{tabular}

advanced cases can occur and treatment options for chemotherapy-refractory cases are not well established. We report a case of response to ICI therapy in a patient with chemotherapy-refractory classic KS. To our knowledge, this is the first report of ICI therapy response in this rare malignancy. In the case presented, the patient was a man that had sex with men, which is a risk factor for HHV-8 acquisition. ${ }^{9}$ The patient's African heritage may also affect HHV-8 subtype and portend for increased severity of disease. ${ }^{10}$

Immunotherapy in this case was selected partly due to the genomic profiling finding of a higher-thananticipated level of somatic mutational burden, and partly due to a general enthusiasm for immunotherapy investigation for virus-related malignancies. Although not a definitive determinant of ICI response, TMB has been well established as predictive biomarker across multiple cancer types, ${ }^{11}$ and has been validated in the same NGS platform used in our patient. ${ }^{6,7}$

Importantly, the genomics of our case differed significantly from those of 8 other cases of $\mathrm{KS}$ that underwent molecular profiling using the same plat- form. Many factors could potentially explain the difference in TMB between the index case and the other $\mathrm{KS}$ cases. First, the sequencing was performed on a biopsy taken when the disease was already at an advanced stage. Therefore, the TMB may partly reflect the acquisition of mutations over the cancer lifetime. We also speculate that different types of KS (classic vs AIDS-associated) might be affected by different TMB. For example, increased genomic "drivers" might be required in classic $\mathrm{KS}$ to compensate for the lack of immunodeficiency-driven oncogenesis that is responsible for tumor initiation in HIV-related KS. To our knowledge, the somatic mutational landscape of classic KS has not been comprehensively evaluated.

Further study is required to investigate whether ICI therapy is more broadly effective in KS. For both HIV-associated and classic KS, several clinical trials are ongoing that will help to answer this question. Based on the described case, immunogenomic profiling to compare classic and HIV-related KS and further clinical study of ICI therapy in classic KS are warranted.

\section{References}

1. Stiller CA, Trama A, Brewster DH, et al. Descriptive epidemiology of Kaposi sarcoma in Europe. Report from the RARECARE project. Cancer Epidemiol 2014;38:670-678.

2. Regnier-Rosencher E, Guillot B, Dupin N. Treatments for classic Kaposi sarcoma: a systematic review of the literature. J Am Acad Dermatol 2013;68:313-331.

3. Di Lorenzo G, Kreuter A, Di Trolio R, et al. Activity and safety of pegylated liposomal doxorubicin as first-line therapy in the treatment of nonvisceral classic Kaposi's sarcoma: a multicenter study. J Invest Dermatol 2008;128:1578-1580.

4. Paydas S, Bagir EK, Deveci MA, Gonlusen G. Clinical and prognostic significance of PD-1 and PD-L1 expression in sarcomas. Med Oncol 2016;33:93.

5. Beldi-Ferchiou A, Lambert M, Dogniaux S, et al. PD-1 mediates functional exhaustion of activated NK cells in patients with Kaposi sarcoma. Oncotarget 2016;7:72961-72977.
6. Goodman AM, Kato S, Bazhenova L, et al. Tumor mutational burden as an independent predictor of response to immunotherapy in diverse cancers. Mol Cancer Ther 2017;16:2598-2608.

7. Johnson DB, Frampton GM, Rioth MJ, et al. Targeted next generation sequencing identifies markers of response to PD-1 blockade. Cancer Immunol Res 2016;4:959-967.

8. Frampton GM, Fichtenholtz A, Otto GA, et al. Development and validation of a clinical cancer genomic profiling test based on massively parallel DNA sequencing. Nat Biotechnol 2013;31:1023-1031.

9. Liu Z, Fang Q, Zuo J, et al. Global epidemiology of human herpesvirus 8 in men who have sex with men: a systematic review and meta-analysis. J Med Virol 2018;90:582-591.

10. Royse KE, El Chaer F, Amirian ES, et al. Disparities in Kaposi sarcoma incidence and survival in the United States: 2000-2013. PLoS One 2017;12:e0182750.

11. Gibney GT, Weiner LM, Atkins MB. Predictive biomarkers for checkpoint inhibitor-based immunotherapy. Lancet Oncol 2016;17:e542-551. 